\title{
Trama trófica de una comunidad de peces en una pradera marina en el Caribe Venezolano
}

\author{
Adriana López-Ordaz ${ }^{1}$, Mario Ortaz ${ }^{1} \&$ José G. Rodriguez-Quintal ${ }^{2}$ \\ 1. Instituto de Biología Experimental, Universidad Central de Venezuela. Apartado 48170 Caracas 1041 A, Venezuela; \\ adri_lopezordaz@yahoo.es,maortaz@hotmail.com \\ 2. Departamento de Biología, Facultad de Ciencias y Tecnología, Universidad de Carabobo. Apartado 2001 Valencia \\ Venezuela; stegastes@hotmail.com
}

Recibido 03-X-2008. Corregido 30-III-2009. Aceptado 27-IV-2009.

\begin{abstract}
Trophic network of a fish community in a seagrass bed in the Venezuelan Caribbean. Seagrass beds are considered important feeding places for a variety of fish species characterized with complex trophic interactions. In this paper we described the trophic network of a fish community inhabiting a seagrass bed in the Venezuelan Caribbean. In addition, a consumption index (CI) for each prey ingested is proposed using two variables: abundance and consumption frequency. Eight samplings (at four time intervals: 6:00, 12:00, 18:00 and 24:00 hours) were done in August 2005 and January 2006. Fifty one fish species in 29 families were captured; Haemulidae was the most abundant. Crustacea was the most important food resource of the 28 food items identified. The relative importance of trophic guilds, considering abundance and taxonomic species richness, was: benthophagous $>$ herbivorous $>$ piscivorous. The features of the trophic network were: 1 ) high proportion of short chains (two links), 2) high number of intermediate species, 3) high consumption of benthos, 4) low CI values and 5) few species with a high number of linkages. Temporal (hourly and monthly) differences were obtained in fish species number (total, intermediate and top), total linkages, connectivity and maximal chain lengths. The connectance did not show significant temporal variation and it was similar to other environments with different species richness. In contrast, the connectivity was lower than the one reported for environments with low species richness. As in other reports, the temporal variation of the trophic parameters and the community structure was different. The values of CI for the different preys were low except for harpacticoid copepods and crustacean larvaes. The cluster value of CI was high for benthos ( $>80 \%)$ and, in general, low for plankton and nekton preys. The seagrass bed studied was an important feeding place where the configuration of trophic network changed in different temporal scales and reflected the dynamic of the feeding relationships this fish community. Rev. Biol. Trop. 57 (4): 963-975. Epub 2009 December 01.
\end{abstract}

Key words: food web, ichthyofauna, seagrass bed, Venezuelan Caribbean.

Las praderas marinas son ambientes muy productivos que poseen una gran abundancia y una alta diversidad de invertebrados y peces (Arrivillaga \& Baltz 1999, Burchmore et al. 1984, Duffy 2006, Nagelkerken et al. 2002, Pollard 1984, Robblee \& Zieman 1984). Se considera que esta alta diversidad se debe a la complejidad estructural del componente vegetal y a la presencia de abundante alimento para los peces, entre el que destaca la fauna macrobentónica (Arrivillaga \& Baltz 1999, Den-Hartog 1976).
Otra característica de estos ambientes es el permanente reemplazo temporal de una fracción de su ictiofauna asociada, dependiendo del estadio de vida de los individuos de las distintas especies que componen la comunidad, así como de la variación estacional en la disponibilidad del alimento, entre otros. Esto se traduce en una serie de interacciones tróficas complejas que producen una trama trófica cuya estructura va cambiando en un ambiente aparentemente estable en cuanto a su condición climática se refiere. 
La descripción y el análisis de las tramas tróficas naturales es un aspecto fundamental de la ecología de comunidades lo cual, además de servir como información de línea de base que permite predecir los posibles cambios sobre una comunidad producto de las alteraciones naturales o antrópicas, puede emplearse, a través de las biomanipulaciones, como una herramienta de restauración de ambientes. Las interacciones alimenticias que definen esta estructura trófica pueden conocerse a través de la cuantificación de una serie de parámetros operativos entre los que se encuentran, el número de especies basales, intermedias y tope, la conectancia, la conectividad y la longitud de las cadenas alimenticias (Briand \& Cohen 1984, Ricklefs \& Miller 2000, Pimm et al. 1991). Sin embargo, ninguno de los parámetros anteriores considera una medida cuantificable de la intensidad de interacción la cual puede variar entre tramas aparentemente parecidas en otros aspectos. La ventaja de estas estimaciones es que permite caracterizar y comparar distintas tramas tróficas, lo cual es una aproximación para descifrar mecanismos de funcionamiento a distintas escalas.

En la región del mar Caribe se ha encontrado que las principales presas de las que depende la trama trófica de la ictiofauna asociada a las praderas marinas son los poliquetos y anfípodos (Brook 1977), otros crustáceos como los copépodos (Heck \& Weinstein 1989, Pollard 1984) y los moluscos (Motta et al. 1995). También se ha encontrado cierta variación espacial en la importancia directa de las fanerógamas como fuente de alimento para una serie de especies de peces asociadas a estos ambientes (Pollard 1984).

En Venezuela son escasos los estudios de tramas tróficas de la ictiofauna tanto continental como la que habita en distintos ambientes marinos incluyendo a las praderas marinas (Díaz 1997, Winemiller 1990, 2005) y la mayoría de ellos han abordado el problema de manera parcial ya que se han centrado en la estimación de las interacciones alimenticias sólo de las especies más abundantes de la comunidad (Allen et al. 2006, González-Cabello 1981, Hug 1984, Ortaz et al. 1996, Rivas et al. 1999).
El objetivo del presente estudio consistió en describir la trama trófica de la ictiofauna asociada a una pradera marina ubicada en la región centro norte del Caribe Venezolano. Para ello, se emplearon los criterios propuestos por Briand \& Cohen (1984) y Pimm et al. (1991). Adicionalmente, se propuso un parámetro descriptor que midió la intensidad de interacción, y se evaluó la variación temporal (mensual e intradiaria) de la trama trófica estudiada.

\section{MATERIALES Y MÉTODOS}

Area de estudio: El Parque Nacional Archipiélago Los Roques (PNALR) se encuentra a 157 kilómetros al norte franco del litoral central Venezolano $\left(11^{\circ} 43^{\prime}-11^{\circ} 58^{\prime} \mathrm{N}\right.$ y $66^{\circ} 35^{\prime}-66^{\circ} 57^{\prime} \mathrm{W}$ ), ocupa un área de $2251 \mathrm{~km}^{2}$ y se extiende $36 \mathrm{~km}$ en dirección oeste-este y $24.6 \mathrm{~km}$ en dirección norte-sur. El clima de la zona es seco y cálido con temperaturas superficiales del agua que oscilan entre $25^{\circ} \mathrm{C}$ y $30^{\circ} \mathrm{C}$, con un mínimo entre enero y febrero y un máximo entre junio y octubre de cada año (Rodríguez 2001). Dentro del PNALR existen praderas de hierbas marinas conformadas por Thalassia testudinum (Banks ex Köning) y, en menor densidad, por Siringodium filiforme (Kütz) y Halodule wrighti (Ashers). Estas praderas constituyen una unidad vegetal de gran valor para las comunidades animales propias de ambientes marinos someros que las utilizan como zona de refugio, desove y alimentación (Villamizar \& Zamarro 2001).

La pradera seleccionada en el presente estudio estuvo ubicada en la zona noreste del cayo Dos Mosquises Sur ( $11^{\circ} 47 \mathrm{~N}$ y $\left.66^{\circ} 53 \mathrm{~W}\right)$, el cual se encuentra al suroeste del PNALR. Esta pradera está constituida por T. testudinum y H. wrighti. Posee un área de $4200 \mathrm{~m}^{2}$, es de poca pendiente y durante el período de muestreo se registró una profundidad máxima de $1.15 \mathrm{~m}$, y una temperatura y salinidad promedio de $30.6^{\circ} \mathrm{C}( \pm 0.9)$ y $36 \%$, respectivamente.

Métodos: Se realizaron dos salidas de campo, en agosto de 2005 y enero de 2006. En cada salida se realizaron cuatro muestreos 
en los siguientes horarios: 6:00, 12:00, 18:00 y 24:00 horas para un total de ocho colectas de campo. Los peces se recolectaron con un chinchorro de playa de $15.4 \mathrm{~m}$ de longitud y $0.7 \mathrm{~cm}$ de abertura de malla. En cada horario se realizaron cuatro caladas. Adicionalmente, se empleó una rastra manual bentónica de $2700 \mathrm{~cm}^{2}$ de área superficial y $1 \mathrm{~mm}$ de abertura de malla, la cual se desplazó séis veces en cada horario de forma aleatoria por un período de 30 segundos. Los peces capturados se preservaron en solución de formol $(10 \% \mathrm{v} / \mathrm{v})$ y posteriormente fueron trasferidos a una solución de etanol $(70 \% \mathrm{v} / \mathrm{v})$ para su preservación definitiva. Para la identificación de los peces se emplearon las claves de Cervigón (1991, 1993, 1994 y 1996) y Fisher (1978). Se analizaron los tractos digestivos de todos los ejemplares capturados en cada horario, sólo en el caso de Haemulon flavolineatum y Harengula humeralis se tomó una submuestra para lo cual se realizaron curvas acumulativas del número de ítems consumidos versus el número de tractos analizados, con lo que se estimó un número significativo de 15 y 20 tractos, respectivamente. Se estimó, en cada ejemplar, la presencia, el número y el peso $(\mathrm{g})$ de los distintos tipos de presas consumidas. Estas variables se agruparon en un índice de importancia relativa (\%IRI) (Ortaz et al. 2006). Se emplearon los criterios de Briand \& Cohen (1984) y Pimm et al. (1991) para estimar el número de especies basales, intermedias y topes, la conectancia trófica $(\mathrm{Ct})$ $(\mathrm{Ct}=\mathrm{L} / \mathrm{S}(\mathrm{S}-1) ; \mathrm{L}=$ número de uniones en la trama, $\mathrm{S}=$ número de especies), la conectividad (C) $(\mathrm{C}=\mathrm{L} / \mathrm{S})$ y la longitud de las cadenas. Se propuso el siguiente índice como una medida de la intensidad de consumo (IC) de cada ítem:

$$
\mathrm{IC}=\left[\left(\mathrm{N}_{\mathrm{i}} / \mathrm{NE}_{\mathrm{i}}\right) \mathrm{x}\left(\mathrm{NSP}_{\mathrm{i}} / \mathrm{NSP}\right)\right]
$$

Donde:

$\mathrm{N}_{\mathrm{i}}$ = número total consumido de la presa tipo "i".

$\mathrm{NE}_{\mathrm{i}}=$ número de estómagos en los que apareció la presa tipo "i".

$\mathrm{NSP}_{\mathrm{i}}=$ número de especies en las que apareció la presa tipo "i".

$\mathrm{NSP}=$ número total de especies analizadas.
El índice de consumo (IC) se estimó para cada presa discreta consumida en cada horario evaluado. Posteriormente y para obtener un estimado de la intensidad de consumo sobre las principales comunidades depredadas se sumaron los distintos valores de IC correspondientes a aquellas presas que pertenecieron a la misma comunidad.

Se realizó un análisis de agrupamiento de los datos de dieta de cada especie estimada con el \%IRI para definir la existencia de gremios o categorías tróficas. Se empleó el método de aglomeración UPGMA y el índice de Morisita como medida de similitud.

\section{RESULTADOS}

Composición global de la dieta de las especies evaluadas e importancia relativa de sus componentes: Se analizaron 51 especies, 42 en agosto/05 y 33 en enero/06, pertenecientes a 29 familias y 11 órdenes. Las familias con la mayor riqueza taxonómica de especies fueron Scaridae (5 especies), Clupeidae, Haemulidae, Labridae, Lutjanidae (cada una con 4 especies), Carangidae, Labrisomidae (cada una con 3 especies), Acanthuridae y Gobiidae (cada una con 2 especies). El resto de las familias (20) estuvieron representadas por una especie. La familia Haemulidae apareció en todos los muestreos realizados y fue la más abundante dentro de la comunidad, siendo Haemulon flavolineatum (Desmarest) la especie mejor representada dentro de la familia. Scaridae fue otra familia importante en términos de abundancia. La mayoría de los ejemplares capturados fueron estadios juveniles o adultos de tallas pequeñas. El 52\% de las especies más abundantes ( $\mathrm{n}=13 \mathrm{spp}$.) no superaron los $50 \mathrm{~mm}$ de longitud estándar (LE). En el 40\% ( $\mathrm{n}=10$ spp.) las tallas variaron entre 50 y $100 \mathrm{~mm}$ de LE y sólo se colectaron 2 especies (Albula vulpes (Linnaeus) y Dasyatis americana (Hildebrand \& Schroeder) con tallas superiores a los $100 \mathrm{~mm}$ (LE>400mm).

Se analizó la dieta de 1014 ejemplares, 546 en agosto/05 y 468 en enero/06, cuyas abundancias horarias variaron en ambos meses. En 
ambos, el mayor porcentaje de estómagos llenos se encontró a las 6:00am (llenura promedio: $84.28 \%$ en agosto/05 y $92.45 \%$ en enero/06) mientras que los menores porcentajes promedio se obtuvieron a las 12:00 en agosto/05 (70\%) y a las 18:00 en enero/06 (57.14\%).

Se identificaron 28 ítems alimenticios, el $88 \%$ de éstos pertenecieron al bentos, el $8 \%$ al necton y el $4 \%$ restante al plancton. El bentos consumido estuvo conformado principalmente por crustáceos, el cual constituyó el grupo más importante en la dieta de la mayoría de las especies. Entre los crustáceos, dominaron los copépodos harpacticoides, los crustáceos decápodos y los anfípodos. El material vegetal bentónico, representado por Halodule wrightii, Thalassia testudinum y algunas macroalgas como Padina sp., Acanthophora sp. y Ulva sp., constituyó una fracción importante (\% IRI>30) en la dieta del $18 \%$ de las especies evaluadas. El necton estuvo conformado por camarones $\mathrm{y}$ peces. Los peces consumidos que pudieron identificarse pertenecieron a las familias Clupeidae (Harengula humeralis (Cuvier) y $H$. clupeola (Cuvier), Engraulidae, Monacanthidae, Muraenidae y Scaridae (Scarus sp.). El plancton estuvo constituido por larvas de crustáceos decápodos y copépodos calanoides.

Si bien se obtuvieron algunas variaciones intrahorarias en el consumo de ciertas presas, como la disminución nocturna en ambos meses en la ingesta de copépodos harpacticoides y peces, no hubo en general una variación en el orden de importancia relativa de las distintas presas consumidas, a excepción de lo obtenido entre las 12:00 y las 18:00 horas (rs de Spearman: $0.251, \mathrm{p}<0.05$ ) y entre las $18: 00 \mathrm{y}$ las 24:00 horas de enero/06 (rs de Spearman: $0.186, \mathrm{p}<0.05)$. Al respecto, el orden general decreciente de importancia de las principales presas (\%IRI) fue el siguiente: copépodos harpacticoides $>$ crustáceos decápodos $>$ larvas de crustáceos $>$ anfípodos $>$ material vegetal $>$ peces.

Descripción de la trama trófica. El 14\% de las especies capturadas fueron consumidores de primer orden (predominantemente herbívoros), y pertenecieron a las familias
Acanthuridae, Hyphorhamphidae, Kiphosidae, Monocanthidae y Scaridae. Este grupo consumió T. testudinum, H. wrightii y macroalgas y en algunas especies se encontraron pequeños crustáceos (copépodos y ostrácodos) en su dieta, aunque en baja proporción. El 80\% fueron consumidores de segundo orden y su dieta estuvo constituida principalmente por crustáceos, anélidos y moluscos y algunas especies consumieron, en menor proporción, material vegetal y peces. Adicionalmente, este grupo fue el mejor representado en la comunidad en términos de abundancia y en él predominó el bentos como recurso alimenticio. Sin embargo, la importancia relativa de los distintos organismos bentónicos varió entre las especies analizadas de modo que se identificaron varios subgrupos en los cuales dominaron los copépodos harpacticoides, los gastrópodos, y los crustáceos decápodos (Fig. 1). Adicionalmente, se identificó otro subgrupo de consumidores secundarios cuyos miembros se alimentaron principalmente de larvas de decápodos y de copépodos calanoides. Los consumidores de segundo orden estuvieron representados por las familias Albulidae, Carangidae, Clupeidae, Dasyatidae, Engraulidae, Gerreidae, Gobiidae, Haemulidae, Labridae, Lutjanidae, Mugilidae, Pomacentridae y Syngnathidae. El 6\% de las especies restantes fueron consumidores de tercer orden (piscívoras) y pertenecieron a las familias Carangidae (Trachinotus goodei (Jordan \& Everman), Lutjanidae (Lutjanus apodus (Walbaum) y Sphyraenidae (Sphyraena barracuda (Walbaum).

En ambos meses y en todos los horarios evaluados el gremio bentófago fue el más abundante en la comunidad mientras que los piscívoros y zooplanctófagos fueron los menos importantes. Los herbívoros ocuparon una posición intermedia entre ambos extremos.

El número total de especies, el número de especies basales, intermedias y topes y el número total de uniones resultó mayor en agosto/05 (Cuadro 1). En este mes los menores valores de estos parámetros se obtuvieron a las 12:00 horas y los mayores a las 18:00 horas. En enero/06 se obtuvo una menor variación 


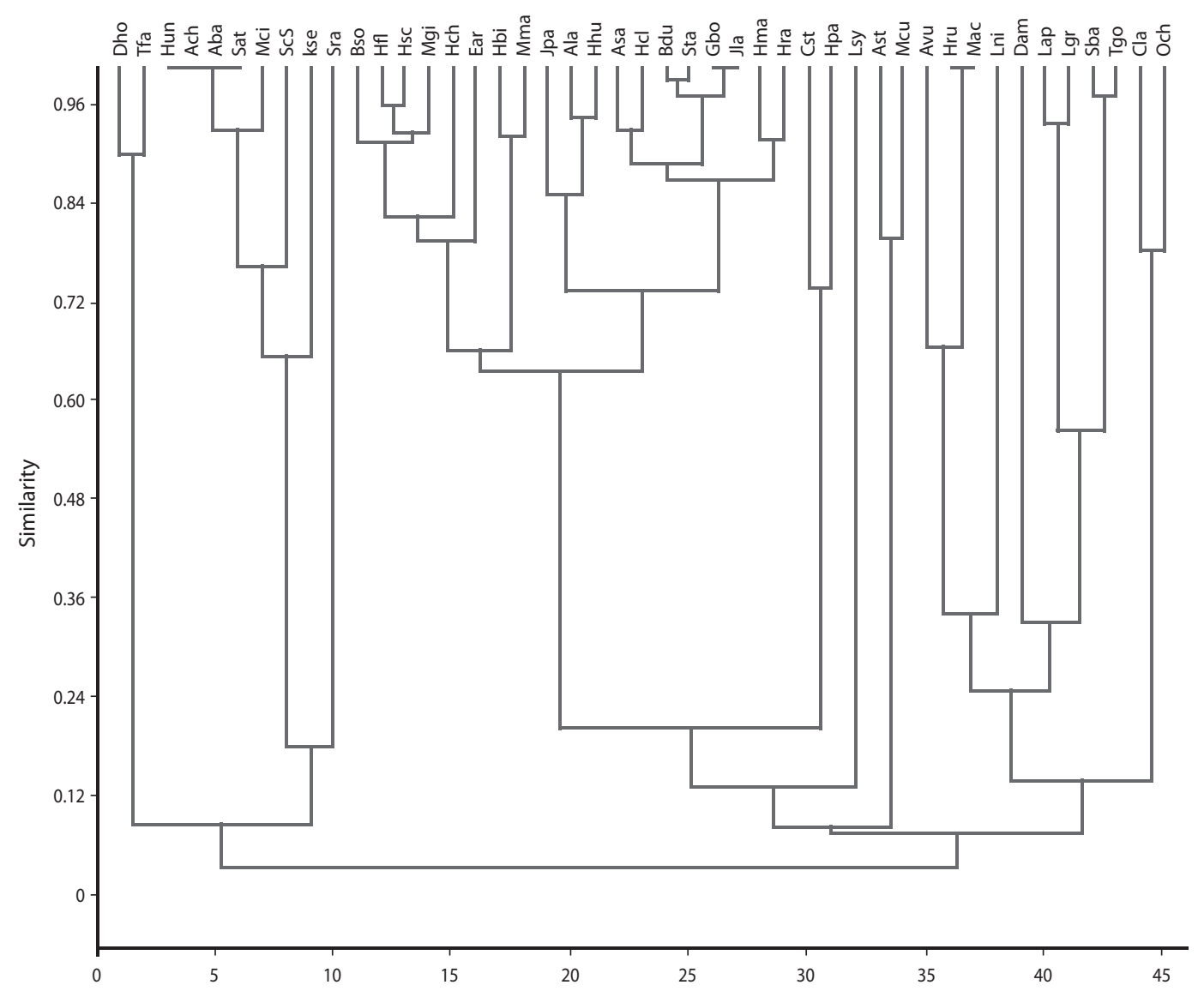

Fig. 1. Análisis de cluster del índice de Morisita calculado con los \% IRI de las presas consumidas. Ach: A. chrirurgus, Aba: A. bahianus Asa: A. saxatilis, Avu: A. vulpes, Ala: A. lamprotaenia, Ast: A. stipes, Bso: B. soporator, Bdu: B. dunckeri, Cla: C. latus, Cst: C. striatus Dam: D. americana, Dho: D. holocanthus, Ear: E. argenteus, Gbo: G. boleosoma, Hbi: $H$. bivittatus, Hcl: H. clupeola, Hch: H. crysargyreum, Hfl: H. flavolineatum, Hhu: H. humeralis, Hma: H. maculipinna, Hpa: H. parrai, Hra: H. radiatus, Hsc: H. sciurus, Hun: H. unifasciatus, Jla: J. lamprotaenia Jpa: J. parvula, Kse: K. sectatrix, Lap: L. apodus, Lgr: L. griseus, Lni: L. negricinctus, Lsy: L. synagris, Mcu: M. curema, Mci: M. ciliatus, Mcu: M. acuminatus, Mgi: M. gilli, Mma: M. macropus, Och: O. chrysurus, Sat: S. atomarium, Scs: Scarus sp, Sra: S. radians, Sta: S. taeniopterus, Spa: Sparisoma sp, Tgo: T. goodei, Tfa: T. falcatus.

Fig. 1. Cluster analysis of Morisita index calculated with \% IRI of preys ingested.

intrahoraria en estos parámetros. A las 6:00 horas se obtuvo el menor número de especies totales, intermedias y de uniones mientras que a las 18:00 estos valores resultaron altos. En todos los horarios, a excepción de las 6:00 horas en agosto/05, dominaron las especies intermedias en la comunidad. En ambos meses fue mayor el número de uniones intermedias
- tope, sin embargo el valor obtenido en agosto/05 resultó mayor. Por el contrario, el número del resto de las uniones (basal-tope e intermedia-intermedia) resultó bajo a excepción de las uniones basales - intermedias (Cuadro 1).

Los valores de $\mathrm{Ct}$ y $\mathrm{C}$ obtenidos a las 6:00 y 18:00 horas fueron mayores en agosto/05 y viceversa para los obtenidos a las 12:00 y 24:00 
CUADRO 1

Parámetros de la trama trófica analizada en cada periodo de muestreo

TABLE 1

Parameters of trophic web analyzed in each sampling period

\begin{tabular}{|c|c|c|c|c|c|c|c|c|c|c|c|}
\hline \multicolumn{6}{|c|}{ Agosto/05 } & \multicolumn{6}{|c|}{ Enero/06 } \\
\hline Parámetro & $6: 00$ & $12: 00$ & $18: 00$ & $24: 00$ & Global & Parámetro & $6: 00$ & $12: 00$ & $18: 00$ & $24: 00$ & Global \\
\hline $\mathrm{S}$ & 45 & 31 & 51 & 41 & 72 & $\mathrm{~S}$ & 32 & 34 & 42 & 34 & 58 \\
\hline EB & 3 & 4 & 5 & 4 & 5 & EB & 3 & 3 & 4 & 3 & 4 \\
\hline EI & 19 & 17 & 26 & 21 & 34 & EI & 15 & 16 & 19 & 19 & 26 \\
\hline ET & 23 & 10 & 20 & 15 & 33 & ET & 14 & 15 & 19 & 12 & 28 \\
\hline $\mathrm{L}$ & 104 & 41 & 120 & 68 & 248 & $\mathrm{~L}$ & 50 & 59 & 59 & 58 & 171 \\
\hline $\mathrm{Ct}$ & 0.054 & 0.047 & 0.048 & 0.043 & 0.048 & $\mathrm{Ct}$ & 0.053 & 0.055 & 0.035 & 0.054 & 0.051 \\
\hline $\mathrm{C}$ & 2.36 & 1.36 & 2.40 & 1.58 & 3.44 & $\mathrm{C}$ & 1.61 & 1.78 & 1.44 & 1.75 & 2.94 \\
\hline $\mathrm{LC}$ & & & & & 4 & $\mathrm{LC}$ & & & & & 3 \\
\hline $\mathrm{N}^{\circ} \mathrm{ba}$ - int & & & & & 24 & & & & & & 21 \\
\hline $\mathrm{N}^{\circ} \mathrm{ba}-\mathrm{top}$ & & & & & 17 & & & & & & 6 \\
\hline $\mathrm{N}^{\circ}$ int- int & & & & & 9 & & & & & & 11 \\
\hline $\mathrm{N}^{\mathrm{o}}$ int- top & & & & & 129 & & & & & & 91 \\
\hline
\end{tabular}

S: número total de especies. EB: número de especies basales. EI: número de especies intermedias. ET: número de especies topes. L: número total de uniones. Ct: Conectancia. C: Conectividad. LC: longitud máxima de cadenas. $\mathrm{N}^{\circ}$ ba - int: número de uniones basales - intermedias. $\mathrm{N}^{\circ} \mathrm{ba}$ - top: número de uniones basales - topes. $\mathrm{N}^{\mathrm{o}}$ int - int: número de uniones intermedias - intermedias. $\mathrm{N}^{\mathrm{o}}$ int - top: número de uniones intermedias - topes.

horas. Sin embargo, la diferencia en los valores de $\mathrm{Ct}$ fue menor que la correspondiente a $\mathrm{C}$. En agosto/05 y a pesar de haber ocurrido una variación intrahoraria importante en el número de especies ( $\Delta$ especies: 20 especies) y de uniones ( $\Delta$ uniones: 79 uniones), los valores de $\mathrm{Ct}$ no difirieron considerablemente y oscilaron entre 0.043 a las 24:00 horas y 0.054 a las 6:00 horas. Por el contrario, la variación intrahoraria de $\mathrm{C}$ fue mayor. En enero/06 se obtuvo una tendencia intrahoraria opuesta ya que la variación de $\mathrm{Ct}$ fue mayor a la obtenida en agosto/05 y menor la correspondiente a C. Contrario a la que se obtuvo en agosto/05, en enero/06 la variación intrahoraria en el número de especies ( $\Delta$ especies: 10 especies) y de uniones fue menor ( $\Delta$ uniones: 9 en enero/06).

En ambos meses y en los distintos horarios evaluados los valores de IC por presa resultaron bajos. En agosto/05, IC fue $<10$ unidades en el $91 \%$ de los casos, fluctuó entre $10<\mathrm{IC}<50$ en el $6 \%$ y fue $>50$ unidades en el $3 \%$ de los casos. En enero/06, IC fue $<10$ unidades en el $86 \%$ de los casos y fluctuó entre $10<\mathrm{IC}<50$ en el $14 \%$. En este mes no se obtuvieron valores de IC $>50$ unidades (Cuadro 2).

Las presas que mostraron los valores más altos de IC fueron los copépodos harpacticoides (presa bentónica) en ambos meses y en todos los horarios evaluados, y las larvas de crustáceos (presa planctónica) en ambos meses pero sólo a las 6:00 horas. En enero/06 los valores horarios de IC correspondientes a los copépodos harpacticoides fueron parecidos (fluctuaron entre 35 y 41 unidades). Por el contrario, en agosto/05 se obtuvo una diferencia horaria, con una mayor intensidad de consumo de esta presa a las 6:00 y 18:00 horas y una relativa baja intensidad de consumo a las 12:00 horas.

Los valores de IC resultaron mayores para las presas pertenecientes al bentos $(>80 \%$ del valor del índice en todos los casos) y, en general, resultaron bajos para las presas del plancton y necton (Cuadro 2). En agosto/05 el consumo 
CUADRO 2

Valores de "IC" para cada presa discreta en cada período de muestreo

TABLE 2

Values of IC for each discrete prey and sampling period

\begin{tabular}{|c|c|c|c|c|c|c|c|c|c|}
\hline \multicolumn{5}{|c|}{ Agosto/05 } & \multicolumn{5}{|c|}{ Enero/06 } \\
\hline Item & $6: 00$ & $12: 00$ & $18: 00$ & $24: 00$ & Item & $6: 00$ & $12: 00$ & $18: 00$ & $24: 00$ \\
\hline $\mathrm{I}_{1}$ & 1.472 & 3.889 & 1.250 & 0.951 & $\mathrm{I}_{1}$ & 2.068 & 1.250 & 1.028 & 7.636 \\
\hline $\mathrm{I}_{2}$ & 0 & 0 & 0 & 0 & $\mathrm{I}_{2}$ & 0 & 0 & 0.222 & 0 \\
\hline $\mathrm{I}_{3}$ & 1.346 & 0.300 & 0.238 & 1.769 & $\mathrm{I}_{3}$ & 0.091 & 0 & 0.111 & 0.233 \\
\hline $\mathrm{I}_{4}$ & 71.343 & 19.564 & 72.380 & 44.498 & $\mathrm{I}_{4}$ & 39.203 & 39.973 & 35.329 & 41.160 \\
\hline $\mathrm{I}_{5}$ & 0.150 & 0 & 0.442 & 0.231 & $\mathrm{I}_{5}$ & 0 & 0 & 0 & 0.100 \\
\hline $\mathrm{I}_{6}$ & 0 & 0 & 1.048 & 0 & $\mathrm{I}_{6}$ & 0 & 0 & 0 & 0 \\
\hline $\mathrm{I}_{7}$ & 0.190 & 0.5 & 0.095 & 0.538 & $\mathrm{I}_{7}$ & 0.364 & 0.067 & 0.444 & 0.2 \\
\hline $\mathrm{I}_{8}$ & 0 & 0.1 & 0.048 & 0.077 & $\mathrm{I}_{8}$ & 0 & 0 & 0 & 0.133 \\
\hline $\mathrm{I}_{9}$ & 0.071 & 0 & 0 & 0 & $\mathrm{I}_{9}$ & 0 & 0 & 0 & 0 \\
\hline $\mathrm{I}_{10}$ & 0 & 0 & 48.980 & 7.692 & $\mathrm{I}_{10}$ & 0 & 0 & 11.222 & 0.1 \\
\hline $\mathrm{I}_{11}$ & 0.714 & 5.7 & 0 & 6.231 & $\mathrm{I}_{11}$ & 0.545 & 0 & 11.919 & 0 \\
\hline $\mathrm{I}_{12}$ & 0 & 0 & 0 & 0 & $\mathrm{I}_{12}$ & 0 & 0 & 0.056 & 0 \\
\hline $\mathrm{I}_{13}$ & 0.4 & 0.3 & 0.238 & 0.154 & $\mathrm{I}_{13}$ & 0.409 & 0.067 & 0.111 & 2.57 \\
\hline $\mathrm{I}_{15}$ & 0 & 0 & 0.048 & 0 & $\mathrm{I}_{15}$ & 0 & 0 & 0 & 1.2 \\
\hline $\mathrm{I}_{16}$ & 0 & 1.5 & 0.911 & 0.538 & $\mathrm{I}_{16}$ & 0 & 0.48 & 0.222 & 0.845 \\
\hline $\mathrm{I}_{17}$ & 3 & 0.2 & 2.377 & 1.202 & $\mathrm{I}_{17}$ & 1.382 & 14.567 & 4.861 & 2.188 \\
\hline $\mathrm{I}_{18}$ & 0 & 0 & 0.048 & 0 & $\mathrm{I}_{18}$ & 0 & 0 & 0 & 0 \\
\hline $\mathrm{I}_{19}$ & 1.070 & 0.775 & 0.825 & 1.090 & $\mathrm{I}_{19}$ & 0.636 & 0.509 & 0.444 & 0.587 \\
\hline $\mathrm{I}_{21}$ & 0.429 & 0 & 0.095 & 0.538 & $\mathrm{I}_{21}$ & 0.091 & 0 & 0.167 & 0.286 \\
\hline $\mathrm{I}_{22}$ & 1.190 & 0.2 & 0.821 & 0.369 & $\mathrm{I}_{22}$ & 0.091 & 0.267 & 0.083 & 0.729 \\
\hline $\mathrm{I}_{23}$ & 0 & 0 & 0.048 & 0.038 & $\mathrm{I}_{23}$ & 0 & 0.767 & 0 & 0 \\
\hline $\mathrm{I}_{24}$ & 0.889 & 0 & 1.071 & 0.077 & $\mathrm{I}_{24}$ & 0.091 & 0 & 0.056 & 0.100 \\
\hline $\mathrm{I}_{25}$ & 0.224 & 0.3 & 0.381 & 0.077 & $\mathrm{I}_{25}$ & 0.273 & 0.770 & 0.111 & 0.150 \\
\hline $\mathrm{I}_{26}$ & 11.106 & 0.525 & 1.238 & 1.108 & $\mathrm{I}_{26}$ & 10.436 & 0.509 & 0.444 & 0.800 \\
\hline Bentos & 83.64 & 34.08 & 134.38 & 67.52 & Bentos & 45.58 & 61.11 & 68.01 & 58.27 \\
\hline Plancton & 11.11 & 0.53 & 1.33 & 1.11 & Plancton & 10.83 & 0.71 & 0.44 & 0.80 \\
\hline Necton & 1.11 & 0.30 & 1.45 & 0.15 & Necton & 0.36 & 0.77 & 0.17 & 0.25 \\
\hline
\end{tabular}

$\mathbf{I}_{1}$ : anfípodo, $\mathbf{I}_{2}$ : bivalvo, $\mathbf{I}_{3}$ : cangrejo, $\mathbf{I}_{\mathbf{4}}$ : copépodo, $\mathbf{I}_{\mathbf{5}}$ : quironómido, $\mathbf{I}_{6}$ : chitón, $\mathbf{I}_{7}$ : ermitaño, $\mathbf{I}_{8}$ : estomatópodo, $\mathbf{I}_{\mathbf{9}}$ : eufásido, $\mathbf{I}_{10}$ : foraminífero, $\mathbf{I}_{\mathbf{1 1}}$ : gastrópodo, $\mathbf{I}_{\mathbf{1 2}}$ : holoturio, $\mathbf{I}_{\mathbf{1 3}}$ : isópodo, $\mathbf{I}_{\mathbf{1 4}}$ : material vegetal, $\mathbf{I}_{15}$ : misidaceo. $\mathbf{I}_{\mathbf{1 6}}$ : opistobranquio, $\mathbf{I}_{\mathbf{1 7}}$ : ostrácodo, $\mathbf{I}_{\mathbf{1 8}}$ : picnogónido, $\mathbf{I}_{\mathbf{1 9}}$ : poliqueto, $\mathbf{I}_{\mathbf{2 0}}$ : resto de crustaceo, $\mathbf{I}_{\mathbf{2 1}}$ : sipuncúlido, $\mathbf{I}_{\mathbf{2 2}}$ : tanaidaceo, $\mathbf{I}_{\mathbf{2 3}}:$ tunicado, $\mathbf{I}_{\mathbf{2 4}}$ : camarón, $\mathbf{I}_{\mathbf{2 5}}$ : pez, $\mathbf{I}_{\mathbf{2 6}}$ : larva de crustaceo.

de bentos (IC) resultó mayor al obtenido en enero/06 en todos los horarios evaluados a excepción de las 12:00 horas y en ambos meses los mayores valores se obtuvieron a las 18:00 horas y los menores a las 12:00 horas en agosto/05 y a las 6:00 horas en enero/06 (Cuadro 2).

Los valores para el plancton sólo resultaron relativamente altos (un poco mayor al 10\% del total) a las 6:00 horas en ambos meses y en el resto de los horarios e igualmente para el necton, estos no representaron más del $2 \%$ del valor total del índice.

El número de cadenas fue mayor en agosto/05 (200 vs 135 en enero/06) así como la máxima longitud de cadena. En agosto/05 se encontraron cadenas constituidas por un 
eslabón $(\mathrm{n}=17)$, dos eslabones $(\mathrm{n}=146)$, tres eslabones $(n=33)$ y cuatro eslabones $(n=4)$. En enero/06 sólo se encontraron cadenas de uno $(n=9)$, dos $(n=97)$ y tres eslabones $(n=29)$. En ambos meses dominaron las cadenas cortas constituidas por dos eslabones (con tres nodos) y su proporción fue parecida en ambos (73\% en agosto/05 y $72 \%$ en enero/06). Igualmente, en ambos meses se obtuvo una relación inversa entre el número de especies y el número de cadenas en las que éstas formaron parte pero en agosto/05 fue mayor la proporción de especies involucradas en un mayor número de cadenas (Fig. 2). Al respecto, en agosto/05, una especie estuvo involucrada en 10 (Malacoctenus
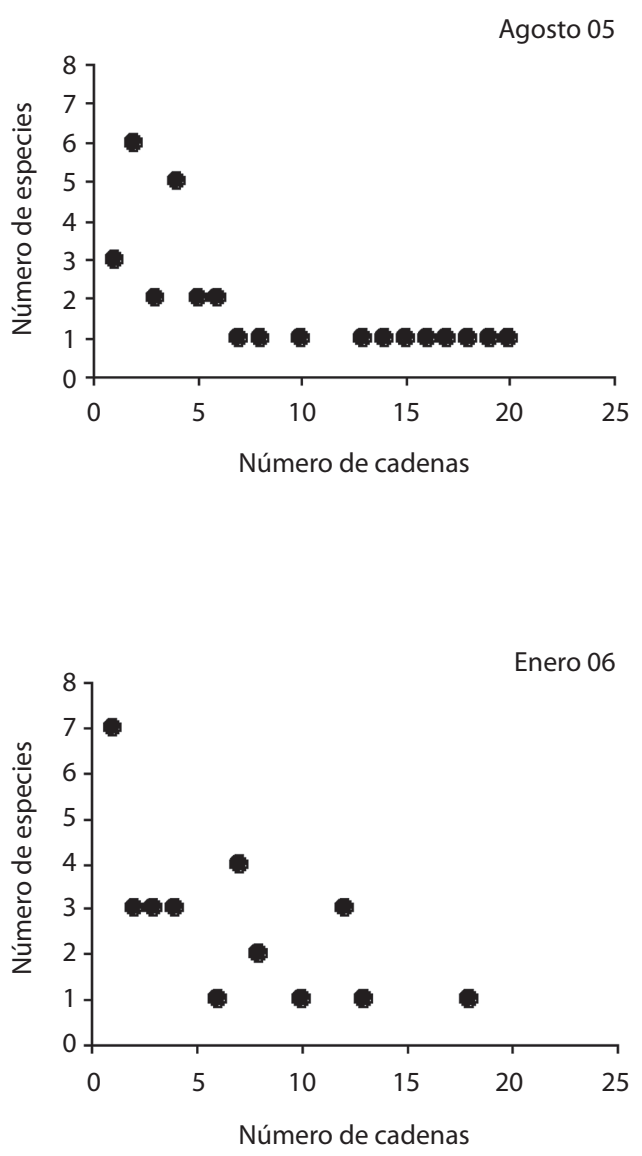

Fig. 2. Relación entre el número de especies y el número de cadenas.

Fig. 2. Relation between species number and chain length. gilli (Steindachner), 13 (Bathygobius soporator (Valeciennes), 14 (Eucinostomus argenteus (Baird \& Girard), 15 (Dasyatis americana), 16 (Trachinotus goodei), 17 (Haemulon crysargyreum (Gunther), 18 (Harengula clupeola), 19 (Haemulon sciurus (Shaw) y 20 cadenas (Haemulon flavolineatum). En enero/06, una especie estuvo involucrada en 10 (Jenkinsia parvula (Jordan \& Evermann), 13 (H. sciurus) y 18 cadenas (L. apodus) y tres especies en 12 cadenas (A. lamprotaenia, H. flavolineatum y L. griseus).

\section{DISCUSIÓN}

Composición global de la dieta de las especies evaluadas e importancia relativa de sus componentes: La complejidad estructural que ofrecen las praderas marinas permite la coexistencia de un gran número de organismos y favorece su uso como sitios de desove, cría y alimentación de muchos juveniles de crustáceos y peces que incluyen especies explotadas comercialmente. En estos ambientes una mayor cobertura y biomasa vegetal favorece una mayor abundancia y diversidad de organismos lo que promueve el desarrollo de tramas tróficas con una estructura compleja (Blaber et al. 1992, Rodríguez 2001).

En la pradera evaluada, la familia Haemulidae resultó muy importante en términos de riqueza de especies y abundancia, lo cual también se ha obtenido en otras localidades en Venezuela (Peña \& Prieto 2001) y en otras áreas del Caribe (Arrivillaga \& Baltz 1999, Nagelkerken et al. 2002). Otro grupo importante, fue Scaridae representado por $S$. radians y $S$. taeniopterus, considerados como característicos de las praderas de T. testudinum (Randall 1965).

El gremio trófico más abundante y diverso dentro de la comunidad evaluada fue el bentófago y además en él se obtuvo la mayor intensidad de consumo. Esto coincidió con lo reportado para otras praderas marinas que indica la importancia de la fauna macrobentónica como la principal fuente de alimento para las especies de peces que habitan estos ambientes y la menor importancia como fuente directa 
de alimento del detritus, el zooplancton y las fanerógamas (Arrivillaga \& Baltz 1999, Brook 1977, Layman \& Silliman 2002, Robertson 1984). El segundo grupo importante fue el de los herbívoros representado por Acanthuridae, Scaridae, Hyporhamphidae, Kyphosidae y Monacanthidae, resultado que corresponde parcialmente con el planteamiento que propone que en el Caribe, la herbivoría es una actividad importante que la ejercen principalmente las especies de las dos primeras familias (Pollard 1984). El gremio zooplanctófago, representado por Clupeidae y Engraulidae, fue menos importante dentro de la comunidad y aunque se alimentó de larvas de crustáceos y copépodos calanoides, algunos de sus integrantes, como H. clupeola y H. humeralis, mostraron una dieta amplia que incluyó componentes del bentos (poliquetos, gastrópodos) y necton (peces). El gremio menos importante fue el de los piscívoros, resultado que coincidió con otros reportes que indican la importancia de las praderas marinas como sitios de refugio y cría de muchas especies de peces, especialmente de los estadios juveniles.

Con el objeto de obtener un estimado de la intensidad de consumo sobre las distintas comunidades depredadas (bentos, necton y plancton) se sumaron los valores de las distintas presas pertenecientes a la misma comunidad lo cual indicó la mayor importancia del bentos como fuente de alimento para la ictiofauna evaluada tal y como se ha reportado en otros ambientes parecidos. En este caso, la sumatoria resultó ser un mejor estimador que el promedio de presas consumidas pertenecientes a la misma comunidad ya que según este último, en algunos de los horarios evaluados, la comunidad del plancton resultó ser la más importante por haber sido intensamente depredada (elevado número de presas) pero por pocos individuos lo que produjo un promedio alto de consumo. Este fue el caso de lo obtenido a las 6:00 horas tanto en agosto/05 $\left(\mathrm{X}_{\text {bentos }}: 2.6\right.$ y $\left.\mathrm{X}_{\text {plancton }}: 3.3\right)$ como en enero/06 $\left(\mathrm{X}_{\text {bentos }}: 1.56 \mathrm{y} \mathrm{X}_{\text {plancton }}: 3.71\right)$.

Descripción de la trama trófica: En la trama trófica evaluada fueron más frecuentes las cadenas cortas (dos eslabones), dominaron las especies intermedias que consumieron fundamentalmente a la macrofauna bentónica, dominaron las uniones intermedias-topes, las intensidades de consumo fueron bajas y un número reducido de especies poseyeron el mayor número de uniones tróficas. Aunque es común que en los ecosistemas marinos dominen las especies intermedias (Dunne et al. 2002), la proporción obtenida fue menor a la reportada para otros ambientes.

El estimador de intensidad de consumo empleado (IC) incluyó 2 medidas importantes, el promedio de presas consumidas y su frecuencia de uso dentro de la comunidad. Sin embargo, sólo se calculó para las presas discretas consumidas de modo que no ponderó la intensidad de consumo de ítems no discretos como el material vegetal el cual puede representar un alimento importante para algunos peces habitantes de las praderas marinas. Al respecto, sería conveniente explorar el comportamiento del índice sustituyendo su primer componente por una medida del peso promedio de cada ítem consumido.

La configuración de la trama trófica evaluada es parecida a la descrita para otros ecosistemas tropicales tanto marinos como dulceacuícolas (Winemiller 2005), de modo que parecieran existir patrones aparentemente comunes en sistemas fisicamente distintos. Sin embargo, existen algunas diferencias. Por ejemplo, se considera a la detritivoría ejercida tanto por invertebrados y peces como un rasgo característico de las tramas tróficas en los grandes ríos tropicales con extensas planicies inundables y que las cadenas cortas, constituidas por el detritus, los peces detritívoros y los peces piscívoros a través de pocas uniones (entre dos y tres), son otro rasgo común. En el caso de la pradera evaluada, si bien las cadenas también fueron cortas, sus constituyentes fueron distintos y estuvieron representados principalmente por el detritus, los invertebrados bentónicos detritívoros y los peces bentófagos, con una mínima representación de peces piscívoros. Otra diferencia es la menor importancia, en los ambientes lóticos tropicales, de las macrófitas 
como fuente directa de alimento mientras que en las praderas marinas, si bien los herbívoros no representan el gremio trófico más importante, éste no es despreciable.

A pesar de las diferencias horarias y mensuales en el número de especies y de uniones, Ct y C variaron poco. Diaz (1997) (datos no publicados) encontró algo parecido en una pradera marina de influencia continental en el Caribe Venezolano. Lo mismo reportó Winemiller (1990) en ríos neotropicales quien planteó que estos parámetros son poco sensibles como detectores de los cambios dinámicos que ocurren a nivel comunitario. Sin embargo, en la trama evaluada se observaron cambios temporales (mensuales) de mayor magnitud en otros parámetros como el número total de cadenas que resultó mayor en agosto/05, mes en el cual también se obtuvo la máxima longitud de cadena, fue mayor el número de uniones intermedias-topes, e incrementó el número total de uniones, la riqueza de especies y el número de cadenas de las que formaron parte de manera simultánea algunas especies. Estos cambios produjeron que la configuración de la trama trófica resultara más compleja en agosto/05 lo cual pudo, en parte, ser consecuencia del aumento en la disponibilidad de presas en este mes en comparación a enero/06 en el que disminuyó tanto la riqueza como la abundancia global de la fauna epibentónica colectada (datos no publicados).

En general, los valores de $\mathrm{C}$ obtenidos son menores que los reportados para otros ambientes marinos, mientras que los valores de $\mathrm{Ct}$ son parecidos a los reportados para ambientes en los que la riqueza de especies es aún mayor (Cuadro 3). Diaz (1997) (datos no publicados) reportó mayores valores de C y $\mathrm{Ct}$ para una pradera marina bajo influencia continental en el Caribe Venezolano y en dicha pradera tanto la riqueza de especies como el número de uniones fue menor a la encontrada en el presente trabajo, lo que refuerza la idea de la poca sensibilidad de estos parámetros ante los cambios intracomunitarios. Adicionalmente, parte de las diferencias encontradas pueden deberse a ciertas limitaciones metodológicas como la dificultad de identificar algunas presas y el empleo simultáneo de distintas resoluciones taxonómicas, lo cual subestimaría el número real de uniones.

Debido a la importancia de la macrofauna bentónica asociada a las praderas marinas para la ictiofauna residente es de esperar que una alteración de la estructura física que ofrece la propia pradera, produzca un impacto negativo sobre la configuración de la trama trófica a través de la reducción de la riqueza taxonómica de especies, de la abundancia, de la intensidad de interacción, de la longitud de las cadenas alimentarias y del número de uniones, entre otros. Una amenaza a la que están sometidas las praderas marinas es la eutrofización y se sabe que, entre los efectos que esta produce, está el cambio en la composición florística de la pradera a favor de las algas epífitas y del fitoplancton y en detrimento del crecimiento

CUADRO 3

Parámetros de tramas tróficas para diferentes ecosistemas marinos

TABLE 3

Food web parameters for differents marine ecosystems

$\begin{array}{lcccl}\text { Localidad } & \mathrm{S} & \mathrm{Ct} & \mathrm{C} & \text { Fuente } \\ \text { Pradera de fanerógamas - Sant Marks } & 48 & 0.10 & 4.60 & \text { Christian \& Luczkovich (1999) } \\ \text { Estuario Ythan } & 124 & 0.06 & 4.76 & \text { Hall \& Rafaelly (1991) } \\ \text { Arrecife coralino (pequeño) } & 50 & 0.22 & 11.1 & \text { Dunne et al. (2002) } \\ \text { Arrecife coralino (grande) } & 245 & 0.05 & 13.8 & \text { Dunne et al. (2002) } \\ \text { Pradera de fanerógamas } & 72 & 0.048 & 3.44 & \text { presente estudio }\end{array}$

S: número de especies. Ct: conectancia. C: conectividad. 
y de la biomasa de las fanerógamas. A su vez, esto puede producir una alteración en la estructura comunitaria de la macrofauna bentónica lo cual puede alterar la estructura trófica de la ictiofauna por la reducción en su complejidad, en la conectividad, conectancia y en la longitud de las cadenas (Duffy 2006, Kemp et al. 1988, Valiela et al. 1997).

La caracterización de las tramas tróficas es una aproximación para describir las relaciones alimentarias de las especies que componen una comunidad y permite la identificación de especies claves con lo cual se pueden predecir los efectos que pueda generar una perturbación dentro de la comunidad. Dunne et al. (2002) sugieren que las comunidades que presentan una mayor conectancia son más robustas y menos sensibles a la pérdida de especies ante una perturbación pero aquellas especies con un mayor número de conexiones actuarían como especies dominantes que tendrían un mayor efecto desestabilizador sobre la comunidad.

Los resultados obtenidos mostraron que en un ambiente con una gran aparente estabilidad climática, como lo es la pradera marina evaluada, ocurrieron cambios en la configuración de la trama trófica a distintas escalas temporales (intradiaria e intermensual), lo que indica lo dinámico de las relaciones alimentarias de las especies que la componen, que en ambientes como el evaluado, no solo dependerán de las variaciones temporales en la disponibilidad del alimento, principalmente de origen bentónico, sino también del efecto de las especies transeúntes las cuales representan una fracción importante dentro de la comunidad.

\section{RESUMEN}

Las praderas marinas son consideradas sitios importantes de alimentación para una variedad de especies de peces, por lo que en estos sistemas son frecuentes las interacciones tróficas complejas. En el presente trabajo se describió la trama trófica de la ictiofauna en una pradera marina ubicada en el Caribe Venezolano. Adicionalmente, se propuso una medida de la intensidad de consumo de cada presa (IC), considerando dos variables: la abundancia y la frecuencia de uso. Se realizaron ocho muestreos (en 4 horarios: 6:00, 12:00, 18:00 y 24:00 horas) en agosto 2005 y enero 2006. Se capturaron 51 especies pertenecientes a 29 familias siendo Haemulidae la más abundante y se identificaron 28 items alimenticios dentro de los cuales, Crustácea fue el más importante como recurso alimenticio. La importancia de los gremios tróficos considerando la abundancia y la riqueza taxonómica de especies fue: bentófago $>$ herbívoro $>$ piscívoro. Las características de la trama trófica fueron las siguientes: 1) una mayor proporción de cadenas cortas (dos eslabones), 2) un elevado número de especies intermedias. 3) un elevado consumo de bentos, 4) unas bajas intensidades de consumo y 5) un reducido número de especies involucradas en el mayor número de uniones tróficas estimadas. Se obtuvieron diferencias temporales (horarias y mensuales) en el número de especies (totales, intermedias y topes), de uniones totales, en la conectividad y en la longitud máxima de cadena. No se observó una variación temporal en la conectancia y sus valores resultaron parecidos a los reportados para otros ambientes marinos con distinta riqueza de especies. Por el contrario, los valores de conectividad resultaron menores a los obtenidos en ambientes con menor riqueza de especies. Como en otros reportes, los parámetros tróficos estimados fueron poco sensibles a los cambios intracomunitarios. IC resultó bajo, a excepción de lo obtenido para los copépodos harpacticoides y las larvas de crustáceos, mientras que, los valores de IC agrupados resultaron altos para las presas del bentos $(>80$ $\%) \mathrm{y}$, en general, bajos para las presas del plancton y necton. La pradera evaluada fue un importante sitio de alimentación en el que cambió la configuración de la trama trófica a distintas escalas temporales, reflejando la dinámica de las relaciones alimentarias de la comunidad de peces.

Palabras clave: trama trófica, ictiofauna, pradera marina, caribe venezolano.

\section{REFERENCIAS}

Allen, T., M. Jiménez \& S. Villafranca. 2006. Estructura y categorías tróficas de peces asociados a praderas de Thalassia testudinum (Hydrochaeritales, Hydrocharitaceae) en el Golfo de Cariaco, Estado Sucre, Venezuela. Invest. Mar. 34: 125-136.

Arrivillaga, A. \& D. Baltz. 1999. Comparison of fishes and macroinvertebrates on seagrass and bare-sand sites on Guatemala Atlantic coast. Bull. Mar. Sci. 65: 301-319.

Blaber, S.J., D.T. Brewer, J.P. Salini, J.D. Kerr \& C. Conacher. 1992. Species composition and biomass of fishes in tropical seagrass at Groote Eylandt, Northern Australia. Est. Coast. Shelf. Sci. 35: 605-620.

Briand, F \& J.E. Cohen. 1984. Community food webs have scale-invariant structure. Nature 307: 264-266.

Brook, I. 1977. Trophic relationships in a seagrass community (Thalassia testudinum), in Card Sound, Florida. 
Fish diets in relation to macrobenthic and cryptic faunal abundance. Trans. Amer. Fish. Soc. 106: 219-227.

Burchmore, J.J., D.A. Pollard \& J.D. Bell. 1984. Community structure and trophic relationships of the fauna of an estuarine Posidonia australis seagrass habitat in Port Hacking, New South Wales. Aquatic Bot. 18: 71-87.

Cervigón, F. 1991. Los peces marinos de Venezuela. Vol. I. Fundación Científica Los Roques, Caracas, Venezuela.

Cervigón, F. 1993. Los peces marinos de Venezuela. Vol. II. Fundación Científica Los Roques, Caracas, Venezuela.

Cervigón, F. 1994. Los peces marinos de Venezuela. Vol. III. Fundación Científica Los Roques, Fundación Polar y Universidad de Oriente, Caracas, Venezuela.

Cervigón, F. 1996. Los peces marinos de Venezuela. Vol. IV. Fundación Científica Los Roques, Fundación Polar y Fundación Museo del Mar, Caracas, Venezuela.

Christian, R. \& J. Luczkovich. 1999. Organizing and understanding a winter's seagrass food web network through effective trophic levels. Ecol. Model. 117: 99-124.

Den-Hartog, C. 1976. Structure, function and classification in seagrass communities, p. 90-122. In: McRoy C y Helfferich C (eds.). Seagrass ecosystem: a scientific perspectiva. U.S. Marine Science, Nueva York, EEUU.

Diaz, Y. 1997. Relaciones tróficas en la ictiofauna asociada a praderas de Thalassia testudinum en el Parque Nacional Morrocoy. Tesis de Licenciatura. Universidad Simón Bolivar, Caracas, Venezuela.

Duffy, JE. 2006. Biodiversity and the functioning of seagrass ecosystems. Mar. Ecol. Progr. Ser. 311: 233-250.

Dunne, J., R. William \& N. Martínez. 2002. Network structure and biodiversity loss in food webs: robustness increase with connectance. Ecol. Lett. 5: 558-567.

Fisher, W. Ed. 1978. FAO species identification sheets for fishery purposes. Western Central Atlantic (fishing area 31, Vols. 1-7.). FAO Fish Circ, Roma, Italia.

González-Cabello, L. 1981. Régimen alimentario del coro-coro Orthopristis ruber (Cuvier 1830) (Pisces: Pomadasidae) en las zonas adyacentes a la isla de
Margarita, Venezuela. Bol. Inst. Oceanog, Univ. Oriente, Venezuela. 20: 25-37.

Hall, S. \& D. Rafaelli. 1991. Food - web patterns: lessons from a species - rich web. J. Anim. Ecol. 60: 826842 .

Heck, K \& M. Weinstein. 1989. Ichthyofauna of seagrass meadows along the caribbean coast of Panamá and in the Gulf of México: composition, structure and community ecology. Mar. Biol. 50: 97-107.

Hug, M. 1984. Alimentación de las dos especies de anchovetas Anchoa hyolepis (Evermamm \& Marsh) y A. lamprotaenia (Hildebrand) Piscs: Engraulidae del Golfo de Cariaco, Venezuela. Bol. Inst. Oceanog, Univ. Oriente, Venezuela. 21: 149-156.

Kemp, WM., W.R. Boynton., L. Murria., C.H.J. Madden., R.L. Wetzel \& F.H. Vera. 1988. Relaciones de luz en praderas de pastos marinos Thalassia testudinum y sus algas epifíticas en un medio ambiente estuarino tropical, p. 193-206. In: Yañez-Arancibia A, Day JW Jr (eds.). Ecología de los ecosistemas costeros en el sureste del golfo de México: la región de la laguna de Términos. UNAM-OEA, Ciudad de México, México.

Layman, C. \& B. Silliman. 2002. Preliminary survey and diet analyses of juvenile fishes o fan estuarine creek on Andros Island, Bahamas. Bull. Mar. Sci. 70: 199210.

Motta, P., K. Clifton, P. Hernández, B. Eggold, S. Giordano \& R. Wilcox. 1995. Feeding relationships among nine species of seagrass fishes of Tampa bay, Florida. Bull. Mar. Sci. 56: 185-200.

Nagelkerken, I., C. Roberts, M. Van Riel, M. Dorenbosch, E. Cocheret, G. Van der Velde, \& P. Nienhuis. 2002. How important are mangroves and seagrass beds for coral-reef fish? The nursery hypothesis tested on an island scale. Mar. Ecol. Prog. Ser. 244: 299-305.

Ortaz, M., M.E. Rocha \& J. Posada. 1996. Food habits of the two sympatric fishes Harengula humeralis and $H$. clupeola (Clupeidae) in the Archipelago Los Roques National Park, Venezuela. Carib. J. Sci. 23: 26-32.

Ortaz, M., P. Borjas Von Bach \& R. Candia. 2006. Alimentación del pez insectívoro neotropical Creagrutus bolivari (Pisces: Characidae) según los métodos gráfico y de importancia relativa. Rev. Biol. Trop. 54: 1227-1239.

Peña, M. \& T. Prieto. 2001. Comunidad de peces en tres praderas de Thalassia testudinum del Golfo de Cariaco, Estado Sucre, Venezuela. Bol. Inst. Oceanogr. Univ. Oriente, Venezuela. 40: 39-48. 
Pimm, S.L., J.H. Lawton \& J.E. Cohen. 1991. Food web patterns and their consequences. Nature. 350: 669674.

Pollard, D.A.1984. A review of ecological studies on seagrass-fish communities, with particular reference to recent studies in Australia. Aquatic Bot. 18: 3-42.

Randall, J.E. 1965. Food habits of ref. fishes of the West Indies. Stud. Trop. Oceanog. 5: 665-847.

Ricklefs, R. \& G. Miller. 2000. Ecology. $4^{\text {th }}$ edition. W.H.Freeman and Company, Nueva York, EEUU. $822 \mathrm{p}$.

Rivas, A., E. Méndez, A. Torres \& L. Martínez. 1999. Hábitos alimenticios de Eucinstomus gula y E. argenteus (Pisces: Gerreidae), Bahía de Mochima, Estado Sucre, Venezuela. Bol. Inst. Oceanogr. Univ. Oriente Venezuela. 38: 91-97.

Robblee, M.B. \& J.C. Zieman. 1984. Diel variation in the fish fauna of a tropical seagrass feeding ground. Bull. Mar. Sci. 34: 335-345.

Robertson, B.A. 1984. A review of ecological studies on seagrass-fish communities, with particular referente studies in Australia. Aquatic Bot 18: 3-42.

Rodríguez, B. 2001. Paisaje y ambiente físico, p. 77-84. In Zamarro J. (eds.). Guía del Parque Nacional
Archipiélago Los Roques. Agencia Española de Cooperación Internacional, Madrid, España.

Valiela I., J. McClelland, J. Hauxwell, P.J. Behr, D. Hersh \& K. Foreman. 1997. Macroalgal blooms in shallow estuaries: control and ecophysiological and ecosystem consequences. Limnol. \& Oceanogr. 42: 1105-1118.

Valiela, I., J. McClelland, J. Hauxwell, P.J. Behr, D. Hersh \& K. Foreman. 1997. Macroalgal blooms in shallow estuarios, sustituir por estuaries: control and ecophysiological and ecosystem consequences. Limnol. \& Oceanogr. 42: 1105-1118

Villamizar, E. \& J. Zamarro. 2001. La vegetación, p. 99-108. In Zamarro J. (eds.). Guía del Parque Nacional Archipiélago Los Roques. Agencia Española de Cooperación Internacional, Madrid, España.

Winemiller, K.O. 1990. Spatial and temporal variation in tropical fish trophic networks. Ecol. Monog. 60: 331-367.

Winemiller, K.O. 2005. Floodplain river food webs: generalization and implications for fisheries management, p. 285-312. In Welcomme RL \& T Petr (eds.). Proceeding of the second international symposium on the management of large rivers for fisheries. Vol. 2. Mekong River Commission, Phnom Penh, Camboya. 
